(C) 2011 IEEE. Personal use of this material is permitted. Permission from IEEE must be obtained for all other uses, in any current or future media, including reprinting/republishing this material for advertising or promotional purposes, creating new collective works, for resale or redistribution to servers or lists, or reuse of any copyrighted component of this work in other works. 


\title{
DOMAIN DECOMPOSITION COMBINED RADIAL BASIS FUNCTION Collocation Method to Solve Transient EdDy CuRRENT Magnetic Problems With Moving Conductors
}

\author{
Guangyuan Yang ${ }^{1}$, Xiaoming Chen ${ }^{1}$, Gang Lei ${ }^{1}$, K.R. Shao ${ }^{1}$, Youguang Guo ${ }^{2}$, Jianguo Zhu ${ }^{2}$, and J.D. Lavers ${ }^{3}$, \\ Fellow, IEEE \\ ${ }^{1}$ College of Electrical \& Electronic Engineering, Huazhong University of Science and Technology, Wuhan, 430074, China \\ ${ }^{2}$ Faculty of Engineering and information technology, University of Technology, Sydney, N.S.W. 2007, Australia \\ ${ }^{3}$ Department of Electrical \& Computer Engineering, University of Toronto, Toronto, ON M5S 3G4, Canada
}

\begin{abstract}
Radial basis function (RBF) collocation method is a kind of pure meshless numerical method and has been applied to solve static and transient electromagnetic problems. Especially, it shows a great advantage in the computation of moving conductor eddy current magnetic problems. To simulate the conductor movement, the field equations are decoupled with superposition principle and solved by time-domain iteration under moving coordinate systems. One problem is that the coefficient matrix of RBF governing equations, which needs to be computed in each iteration step, is full. As the number of RBF nodes increases, the computational capacity will grow rapidly. The domain decomposition method (DDM), which divides the solving domain into several sub-domains, could be conveniently combined with RBF collocation method. This paper first applies DDM combined RBF collocation method to compute transient eddy current magnetic field problems with moving conductors. With this novel method, the iteration only proceeds in the sub-domains containing conductors. And the magnetic field in the sub-domains without conductors needs to be computed just once before the iteration. The dimension of the coefficient matrix computed in the iteration is only determined by the number of nodes in the corresponding sub-domains and on the interfaces. An engineering problem is computed to show that the DDM combined RBF collocation method is much more efficient than the normal one.
\end{abstract}

Index Terms — domain decomposition method, eddy current magnetic field, moving conductor, radial basis function

\section{INTRODUCTION}

$\mathrm{T}$ he research on the computation of transient eddy current magnetic field problems with moving conductors has always been attractive in computational electromagnetics [1][6]. Because of the movement of the conductor, the numerical model needs to be modified during the computation. The traditional mesh-based method, for example, the finite element method (FEM), is difficult to deal with this model reconfiguration. One approach is to refresh the meshes in the solving domain in each iteration step according to the conductor movement. But it is not economic when the model is complex. Some improved methods are developed to avoid the mesh refreshment such as mesh coupling method (MCM) [4], finite element-boundary element method (FEM-BEM) [5] and composite grid method (CGM) [6]. With these methods, the moving and static parts of the model are constructed under separate mesh systems and coupled together during computation. A great computational capacity is still required to deal with the model combination.

Radial basis function (RBF) collocation method is a newly developed meshless method and has been applied to solve static and transient electromagnetic problems [7]. As a kind of pure meshless method, the primary functions are constructed on nodes established in the whole solving domain and on the boundaries instead of meshes and the governing equations are obtained through using collocation on each nodes. This method has been proved effective to solve moving conductor eddy current magnetic problems by time-domain iteration under moving coordinate systems [8]. However, the coefficient matrix of the RBF collocation governing equation, which needs to be solved in each iteration step, is full. As the number of RBF node increases, the computational capacity will grow rapidly. Because of this limitation, it is difficult to apply RBF collocation method to solve 3-D problems or electromagnetic systems with complex shapes.

The domain decomposition method (DDM) is a useful tool to divide the solving domain into several sub-domains. This method has been introduced into RBF collocation method to compute electrostatic problems with multi-materials [9]. With the DDM combined RBF collocation method, a sparse coefficient matrix like the FEM could be obtained. Moreover, in the transient eddy current problems, only the magnetic field on nodes belonging to the conductor area is governed by the convective diffusion equation. The field on the other nodes still satisfies the Poisson equation as static problems. Therefore, the iteration could only proceed in the sub-domains with conductor parts. So the dimension of the coefficient matrix computed in each iteration step could be greatly reduced by setting more sub-domains and limiting the node number in the sub-domains with conductor parts.

To examine this method, an electromagnetic launcher system is computed and the computational capacity is analyzed. Through the numerical example, we could see that DDM combined RBF collocation method is much more efficient than the normal one.

\footnotetext{
Manuscript received January 1, 2008 (date on which paper was submitted for review). Corresponding author: Guangyuan Yang (e-mail: kkyanggy@126.com).

Digital Object Identifier inserted by IEEE
} 


\section{RBF COLLOCATION GOVERNING EQUATIONS OF MOVING Conductor Eddy CuRrent MAgnetic Field Problems}

Without loss of generality, consider a 2-D magnetic field boundary value problem as follows. Scalars $A$ and $J_{s}$ express the z-axis direction component of magnetic vector potential and the excitation current density. The solving domain $\Omega$ with a boundary $\xi$ is assumed homogeneous and isotropic. In $\Omega$, a nonmagnetic conductor area $\Omega_{e}$ is moving with a speed $\boldsymbol{V}$ and the nonconducting area is denoted by $\Omega_{h}=\bar{\Omega}_{e} \cap \Omega$. The conductivity $\sigma$ and the permeability $\mu$ are assumed constant. The governing equations of $A$ are:

$$
\begin{array}{ll}
\sigma \mu \frac{\partial A}{\partial t}-\nabla^{2} A+\sigma \mu\left(V_{y} \frac{\partial A}{\partial y}+V_{x} \frac{\partial A}{\partial x}\right)=\mu J_{s} & \text { in } \Omega_{e} \\
\nabla^{2} A=-\mu J_{s} & \text { in } \Omega_{h} \\
B(A)=g & \text { on } \xi
\end{array}
$$

where $B(\cdot)$ means a boundary operator. On Dirichlet boundary $B(A)=A$ and on Neumann boundary $B(A)=\partial A / \partial x$ or $\partial A / \partial y, g$ is a known function defined on $\xi$.

To solve (1), we sequentially set $N_{I}+N_{B}=N$ collocation nodes in $\Omega$ and on $\xi$ respectively and obtain the RBF approximating form of $A$ as:

$$
A(\boldsymbol{x}, t)=\sum_{i=1}^{N} a_{i}(t) \varphi\left(\left\|\boldsymbol{x}-\boldsymbol{x}_{i}\right\|\right)=\boldsymbol{\varphi}^{T}(\boldsymbol{x}) \boldsymbol{a}(t)
$$

where $\varphi\left(\left\|\boldsymbol{x}-\boldsymbol{x}_{i}\right\|\right)$ is the RBF centered at the node with a coordinate $\boldsymbol{x}_{i}=\left(x_{i}, y_{i}\right)$ and $\boldsymbol{\varphi}(\boldsymbol{x})$ is the vector form, $\|\cdot\|$ means the Euclid norm, $\boldsymbol{a}(t)$ is the unknown coefficient vector. The RBF collocation iterative scheme to solve (1) could be seen in [8]. To simulate the conductor movement, $A$ is regarded as a superposition of two fields $A_{s}$ and $A_{e}$ approximated by separate RBFs in moving coordinate systems as:

$$
A=A_{s}+A_{e}=\boldsymbol{\varphi}_{s}^{T}\left(\boldsymbol{x}_{s}\right) \boldsymbol{a}_{s}(t)+\boldsymbol{\varphi}_{e}^{T}\left(\boldsymbol{x}_{\boldsymbol{e}}\right) \boldsymbol{a}_{e}(t)
$$

where the subscripts $s$ and $e$ mean the excitation current and eddy current respectively. $A_{s}$ could be computed as static magnetic problems and we have a linear proportional relationship between $\boldsymbol{a}_{s}$ and $J_{s}$ [10]. So in the following analysis, $A_{s}$ is treated as known parameter in the iteration. And the computational capacity is mainly consumed to solve $A_{e}$. The node number of $\boldsymbol{\varphi}_{e}$ is denoted by $N_{e}=N_{e I}+N_{e B}$. Among the $N_{e I}$ nodes in $\Omega$, we assume that nodes numbered by 1 to $N_{e E}$ and $N_{e E}+1$ to $N_{e I}$ belong to $\Omega_{e}$ and $\Omega_{h}$ respectively. The iterative scheme of $\boldsymbol{a}_{e}$ could be written as:

$$
\begin{array}{lr}
L^{+}\left(\boldsymbol{\varphi}_{e}{ }^{T}\left(\boldsymbol{x}_{e i}\right)\right) \boldsymbol{a}_{e}{ }^{k+1}=L^{-}\left(\boldsymbol{\varphi}_{e}{ }^{T}\left(\boldsymbol{x}_{e i}\right)\right) \boldsymbol{a}_{e}{ }^{k}+b_{i}, & i=1, \cdots N_{e E} \\
\nabla^{2}\left(\boldsymbol{\varphi}_{e}{ }^{T}\left(\boldsymbol{x}_{e i}\right)\right) \boldsymbol{a}_{e}{ }^{k+1}=0, & i=N_{e E}+1 \cdots N_{e I} \\
B\left(\boldsymbol{\varphi}_{e}{ }^{T}\left(\boldsymbol{x}_{e i}\right)\right) \boldsymbol{a}_{e}{ }^{k+1}=g\left(\boldsymbol{x}_{e i}\right), & i=N_{e I}+1 \cdots N_{e}
\end{array}
$$

where $k$ is the number of iteration steps. $L_{e}(\cdot)$ means a linear operator and has an expression as:

$$
L_{e}^{ \pm}(\cdot)=\frac{\sigma \mu}{\Delta t} \cdot(\cdot) \mp \frac{1}{2} \nabla^{2}(\cdot) \pm \sigma \mu\left(V_{x}^{k} \frac{\partial}{\partial x}(\cdot)+V_{y}^{k} \frac{\partial}{\partial y}(\cdot)\right)
$$

where $\Delta t$ is the time interval. According to (3), the component $L^{-}\left(\boldsymbol{\varphi}_{e}{ }^{T}\left(\boldsymbol{x}_{e i}\right)\right) \boldsymbol{a}_{e}{ }^{k}$ could be rewritten as $L^{-}\left(A_{e i}{ }^{k}\right)$. $b_{i}$ is a known quantity which could be solved through $A_{s}$ as:

$$
\begin{aligned}
& b_{i}=-\sigma \mu\left(V_{x}{ }^{k} \frac{\partial A_{s}^{k+1}\left(\boldsymbol{x}_{e i}^{\prime}\right)}{\partial x}\right.\left.+V_{y}{ }^{k} \frac{\partial A_{s}^{k+1}\left(\boldsymbol{x}_{e i}^{\prime}\right)}{\partial y}\right) \\
&-\left(\frac{\sigma \mu}{\Delta t}\left(A_{s}^{k+1}\left(\boldsymbol{x}_{e i}^{\prime}\right)-A_{s}^{k}\left(\boldsymbol{x}_{e i}^{\prime}\right)\right)\right.
\end{aligned}
$$

where $\boldsymbol{x}_{e i}^{\prime}$ means the coordinate of node $N_{e i}$ in the coordinate system of $A_{s}$. There are $N_{e}$ unknowns in (4) and the coefficient matrix is full. As $N_{e}$ increases, the computation to solve (4) will become quite time-consuming.

\section{DDM COMBINED RBF COLLOCATION METHOD}

The main idea of DDM is to divide the solving domain $\Omega$ into several sub-domains in which the field could be computed simultaneity. This method has been introduced into RBF collocation method to solve multi-materials electrostatic problems [9].

Without loss of generality, we divide $\Omega$ into two subdomains $\Omega_{1}$ and $\Omega_{2}$. The conductor area $\Omega_{e}$ belongs to $\Omega_{1}$. The boundary $\xi$ is also divided into two parts $\xi_{1}$ and $\xi_{2}$ as the boundaries of $\Omega_{1}$ and $\Omega_{2}$ respectively. The magnetic field $A_{e 1}$ and $A_{e 2}$ in each sub-domain satisfy (4). The interface between $\Omega_{1}$ and $\Omega_{2}$ is denoted by $\Gamma$ and we have:
$A_{e 1}-A_{e 2}=0$
on $\Gamma$
$\partial A_{e 1} / \partial n-\partial A_{e 2} / \partial n=0$
on $\Gamma$

where $n$ is the normal direction of $\Gamma$.

Then we use the DDM combined RBF collocation model to solve (4). First, we sequentially set two groups of RBF nodes in each sub-domain and on their boundaries respectively. Their numbers are denoted by $N_{e 1}$ and $N_{e 2}$. Among these $N_{e 1}$ and $N_{e 2}$ nodes, the last $N_{e L 1}$ and $N_{e L 2}$ nodes are respectively set on $\Gamma$. The magnetic fields approximated by these two groups of RBFs like (2) are denoted by $A_{e 1}^{\prime}$ and $A_{e 2}^{\prime}$. Then we set another $N_{\Gamma}$ nodes on $\Gamma$ and use $\boldsymbol{x}_{\Gamma}$ to denote their coordinates. Apparently, this group of nodes could be seen in both sub-domains. So we define functions $\lambda_{1}$ and $\lambda_{2}$ on them and let:

$A_{e 1}=A_{e 1}^{\prime}+\lambda_{1}, A_{e 2}=A_{e 2}^{\prime}+\lambda_{2}$

$A_{e 1}^{\prime}$ and $A_{e 2}^{\prime}$ could be solved through:

$L^{+}\left(\boldsymbol{\varphi}_{e 1}{ }^{T}\left(\boldsymbol{x}_{e 11}\right)\right) \boldsymbol{a}_{e 1}{ }^{k+1}=L^{-}\left(A_{e 1 i}{ }^{k}\right)+b_{i}, \quad i=1 \cdots N_{e E 1}$

$\nabla^{2}\left(\boldsymbol{\varphi}_{e 1}^{T}\left(\boldsymbol{x}_{e 1 i}\right)\right) \boldsymbol{a}_{e 1}{ }^{k+1}=0, \quad i=N_{e E 1}+1 \cdots N_{e l 1}$

$B\left(\boldsymbol{\varphi}_{e 1}{ }^{T}\left(\boldsymbol{x}_{e 1 i}\right)\right) \boldsymbol{a}_{e 1}{ }^{k+1}=g\left(\boldsymbol{x}_{e 1 i}\right), \quad i=N_{e l 1}+1 \cdots N_{e 1}-N_{e L 1}$ (9c)

$B\left(\boldsymbol{\varphi}_{e 1}^{T}\left(\boldsymbol{x}_{e 1 i}\right)\right) \boldsymbol{a}_{e 1}{ }^{k+1}=0$, $i=N_{e 1}-N_{e L 1}+1 \cdots N_{e 1}$

$\nabla^{2}\left(\boldsymbol{\varphi}_{e 2}{ }^{T}\left(\boldsymbol{x}_{e 2 i}\right)\right) \boldsymbol{a}_{e 2}{ }^{k+1}=0$, $i=1 \cdots N_{e I 2}$

$B\left(\boldsymbol{\varphi}_{e 2}{ }^{T}\left(\boldsymbol{x}_{e 2 i}\right)\right) \boldsymbol{a}_{e 2}{ }^{k+1}=g\left(\boldsymbol{x}_{e 2 i}\right), \quad i=N_{e I 2}+1 \cdots N_{e 2}-N_{e L 2}$

$B\left(\boldsymbol{\varphi}_{e 2}{ }^{T}\left(\boldsymbol{x}_{e 2 i}\right)\right) \boldsymbol{a}_{e 2}{ }^{k+1}=0$,

$i=N_{e 2}-N_{e L 2}+1 \cdots N_{e 2}$ 
Using the subscript $p=1$ or 2 to denote the corresponding subdomain, the expression of component $\lambda_{p}{ }^{k+1}$ is:

$$
\lambda_{p}^{k+1}=\sum_{m=1}^{N_{\Gamma}} a_{\Gamma m}^{k+1} \psi_{p m}\left(\boldsymbol{x}, \boldsymbol{x}_{\Gamma m}\right)=\boldsymbol{\psi}_{p}{ }^{T} \boldsymbol{a}_{\Gamma}^{k+1}, \quad \boldsymbol{x} \in \Omega_{p}
$$

The primary function $\psi_{p m}$ is constructed on node $\boldsymbol{x}_{\Gamma m}$ and expressed by the RBFs in the sub-domain $\Omega_{p}$ as:

$\psi_{p m}\left(\boldsymbol{x}, \boldsymbol{x}_{\Gamma m}\right)=\sum_{j=1}^{N_{e p}} \beta_{p j} \varphi_{e p}\left(\left\|\boldsymbol{x}-\boldsymbol{x}_{e p j}\right\|\right), \quad \boldsymbol{x} \in \Omega_{p}$

From (11)-(12), we could see that the function $\psi_{p m}$ has a similar property to the RBF. The coefficient $\boldsymbol{\beta}_{p}$ could be solved through:

$$
\begin{array}{lc}
\sum_{j=1}^{N_{e 1}} \beta_{1 j} L^{+}\left(\varphi_{e 1}\left(\left\|\boldsymbol{x}_{e 1 i}-\boldsymbol{x}_{e 1 j}\right\|\right)\right)=0, & i=1 \cdots N_{e E 1} \\
\sum_{j=1}^{N_{e 1}} \beta_{1 j} \nabla^{2}\left(\varphi_{e 1}\left(\left\|\boldsymbol{x}_{e 1 i}-\boldsymbol{x}_{e 1 j}\right\|\right)\right)=0, & i=N_{e E 1}+1 \cdots N_{e I 1} \\
\sum_{j=1}^{N_{e 1}} \beta_{1 j} B\left(\varphi_{e 1}\left(\left\|\boldsymbol{x}_{e 1 i}-\boldsymbol{x}_{e 1 j}\right\|\right)\right)=0, & i=N_{e l 1}+1 \cdots N_{e 1}-N_{e L 1}
\end{array}
$$$$
\sum_{j=1}^{N_{e 1}} \beta_{1 j} \varphi_{e 1}\left(\left\|\boldsymbol{x}_{e 1 i}-\boldsymbol{x}_{e 1 j}\right\|\right)=\varphi_{e 1}\left(\left\|\boldsymbol{x}_{\Gamma m}-\boldsymbol{x}_{e 1 i}\right\|\right),
$$$$
i=N_{e 1}-N_{e L 1}+1 \cdots N_{e 1}
$$$$
\sum_{j=1}^{N_{e 2}} \beta_{2 j} \nabla^{2}\left(\varphi_{e 2}\left(\left\|\boldsymbol{x}_{e 2 i}-\boldsymbol{x}_{e 2 j}\right\|\right)\right)=0, \quad i=1 \cdots N_{e I 2}
$$$$
\sum_{j=1}^{N_{e 2}} \beta_{2 j} B\left(\varphi_{e 2}\left(\left\|\boldsymbol{x}_{e 2 i}-\boldsymbol{x}_{e 2 j}\right\|\right)\right)=0, \quad i=N_{e I 2}+1 \cdots N_{e 2}-N_{e L 2}
$$$$
\sum_{j=1}^{N_{e 2}} \beta_{2 j} \varphi_{e 2}\left(\left\|\boldsymbol{x}_{e 2 i}-\boldsymbol{x}_{e 2 j}\right\|\right)=\varphi_{e 2}\left(\left\|\boldsymbol{x}_{\Gamma m}-\boldsymbol{x}_{e 2 i}\right\|\right),
$$$$
i=N_{e 2}-N_{e L 2}+1 \cdots N_{e 2}
$$

After we obtained the function $\psi_{p}$ on each node $\boldsymbol{x}_{\Gamma}, \boldsymbol{a}_{\Gamma}^{k+1}$ could be solved through substituting (8)-(12) into (7b) as:

$$
\begin{aligned}
\sum_{j=1}^{N_{\Gamma}} a_{\Gamma j}^{k+1}\left(\frac{\partial \psi_{1 j}}{\partial n}\left(\boldsymbol{x}_{\Gamma i}, \boldsymbol{x}_{\Gamma j}\right)-\frac{\partial \psi_{2 j}}{\partial n}\left(\boldsymbol{x}_{\Gamma i}, \boldsymbol{x}_{\Gamma j}\right)\right) \\
=\sum_{j=1}^{N_{e 2}} a_{e 2 j}^{k+1} \frac{\partial \varphi_{e 2}}{\partial n}\left(\left\|\boldsymbol{x}_{\Gamma i}-\boldsymbol{x}_{e 2 j}\right\|\right)-\sum_{j=1}^{N_{e 1}} a_{e 1 j}^{k+1} \frac{\partial \varphi_{e 1}}{\partial n}\left(\left\|\boldsymbol{x}_{\Gamma i}-\boldsymbol{x}_{e 1 j}\right\|\right), \\
i=1,2, \cdots N_{\Gamma}
\end{aligned}
$$

According to (8), the field $A_{e}^{k+1}$ could be expressed as:

$$
A_{e}^{k+1}(\boldsymbol{x})=\boldsymbol{\varphi}_{e p}{ }^{T}(\boldsymbol{x}) \boldsymbol{a}_{e p}^{k+1}+\boldsymbol{\psi}_{p}{ }^{T}(\boldsymbol{x}) \boldsymbol{a}_{\Gamma}^{k+1}, \quad \boldsymbol{x} \in \Omega_{p}
$$

Consider the situation that $W$ sub-domains $\Omega_{1} \cdots \Omega_{W}$ with $M$ interfaces $\Gamma_{1} \cdots \Gamma_{M}$ are defined in $\Omega$. We still could use (9) or (10) to compute $A_{e p}^{\prime}$ according to the condition that whether there is conductor area in $\Omega_{p}$. However, because (15) must be applied on all the interfaces, the number of nodes to construct $\lambda_{p}$ should be equal to the sum of nodes number on each interface as: $N_{\Gamma}=\sum_{m=1}^{M} N_{m \Gamma}$ and $\boldsymbol{a}_{\Gamma}$ should be rewritten as:
$\boldsymbol{a}_{\Gamma}^{T}=\left[\boldsymbol{a}_{1 \Gamma}{ }^{T}, \boldsymbol{a}_{2 \Gamma}{ }^{T}, \cdots, \boldsymbol{a}_{M \Gamma}{ }^{T}\right]$

One sub-domain, for example, $\Omega_{1}$ may have several interfaces between other sub-domains. On these interface, we should compute the function $\psi_{1}$ on each nodes. And on the interfaces which do not belong to $\Omega_{1}$, we have $\psi_{1}=0$. This means that the coefficient matrix of (15) to solve $\boldsymbol{a}_{\Gamma}$ is a sparse one which is easy to save and compute.

Then we analyze the computational capacity in each iteration step. From (10), we see that $A_{e}^{\prime}$ in sub-domains without conductors could be treated as static problems and computed just once before the iteration like $A_{s}$. In the subdomains with conductors, the governing equation (9) has a similar form to (4) and need to be computed in each step. Equation (9) has a $N_{e p} \times N_{e p}$ full coefficient matrix. Fortunately, we could reduce $N_{e p}$ by dividing more sub-domains. Because $\boldsymbol{\psi}_{p}$ does not change during the iteration, $\boldsymbol{\beta}_{p}$ could also be computed and saved beforehand. As mentioned above, equation (16) has a $N_{\Gamma} \times N_{\Gamma}$ sparse coefficient matrix and need to be solved in each step. Using $C_{e p}$ and $C_{\Gamma}$ to express the computational capacity of solving (9) in $\Omega_{p}$ and solving (16) respectively, the computational capacity of DDM combined RBF collocation method to solve (4) in one iteration step is:

$C_{t}=C_{\Gamma}+\sum_{p=1}^{P} C_{e p}, \quad P \leq W$

where we assume that the serial numbers of sub-domains with conductors are $1,2, \ldots, P$.

\section{Numerical EXAmPLE AND Result ANALysis}

A tubular electromagnetic launcher system (coil gun) is computed to examine the presented method. This is a typical moving conductor eddy current magnetic problem.
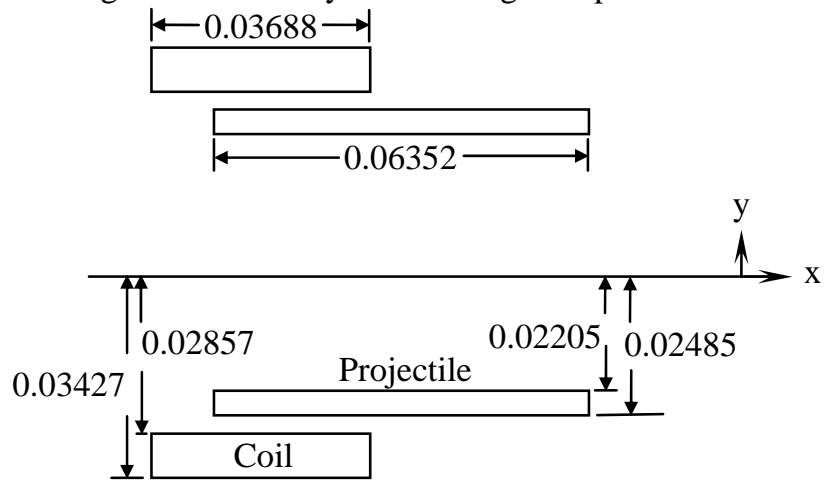

Fig. 1. Dimensions of the 2-D axisymmetric model of electromagnetic launcher system. (Unit: m)

Fig. 1 shows the 2-D axisymmetric model of the system. The excitation current, which is illustrated in Fig. 2, is assumed homogeneous in the coil section and the turn number of the coil is 60 . Because of the time-variable magnetic field, eddy current will be generated in the hollow-cylinder shaped conducting projectile. And the projectile will be accelerated to a high speed in a short time by the magnetic force. At $t=0$, the 
left edge of the projectile is at the middle place of the coil, which refers to the plane $x=0$. The detailed $R B F$ collocation model to compute magnetic force and motion parameters of the conductor could be seen in [8].

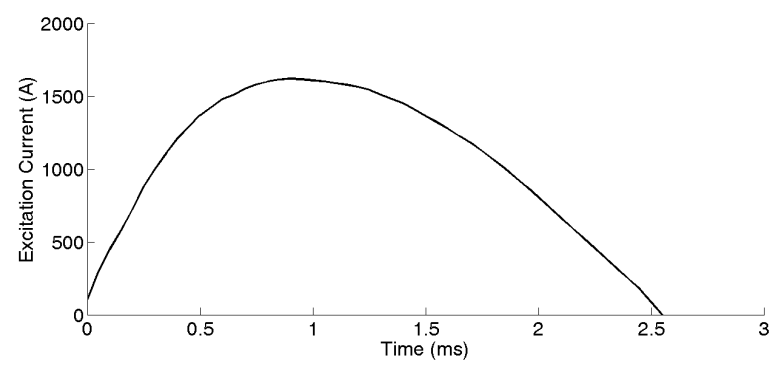

Fig. 2. Variation curve of excitation current in the coil.

In this paper, the eddy current magnetic field $A_{e}$ defined by (3) is solved by the normal RBF and DDM combined RBF collocation method respectively. Because of the symmetry, the solving domain is defined as: $-0.06 \leq x \leq 0.12,0 \leq y \leq 0.06$. In the normal RBF model, the number of nodes is $61 \times 41=2501$. And we divide the solving domain into 6 subdomains as shown in Fig. 3. The projectile section is in one sub-domain with $23 \times 21=483$ nodes and there are 66 nodes on all the interfaces.

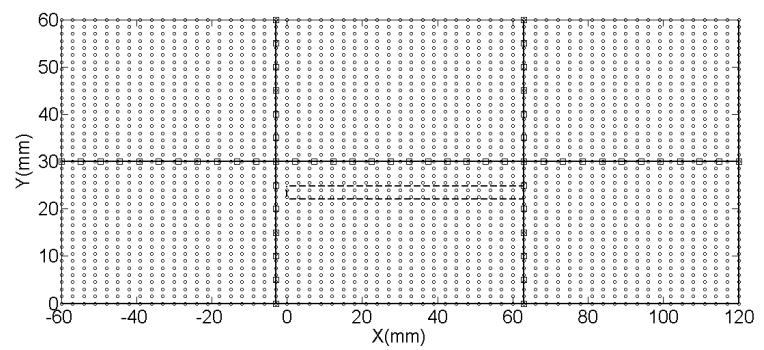

Fig. 3. Nodes distribution in DDM combined RBF model. The round and square markers refer to the nodes in the sub-domains and on the interfaces respectively. The projectile section is expressed by dashed lines.

The speed of the projectile calculated by normal and DDM combined RBF collocation method is shown in Fig. 4. The experiment data and numerical result calculated by FEM [11] are also illustrated. Fig. 4 shows that a proper result could be obtained by both methods. However, the computing time of normal and DDM combined method in one iteration step is $63.7 \mathrm{~s}$ and $0.632 \mathrm{~s}$ respectively.

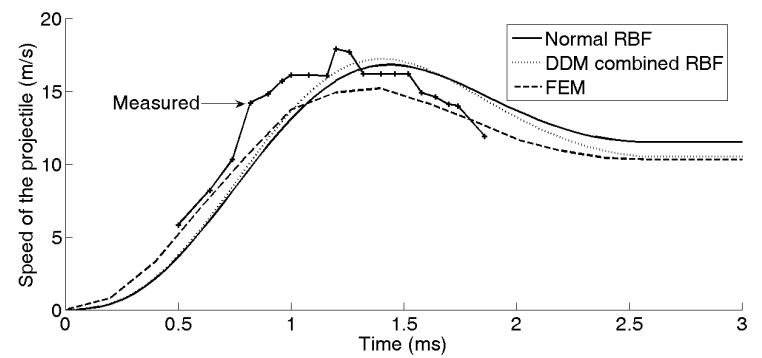

Fig. 4. Measured and calculated speeds of the projectile versus time.

Fig. 5 shows the isopotential lines of $A_{e}$ at $t=0.5 \mathrm{~ms}$ calculated by the DDM combined method. From Fig. 5, we could see that the field near the interfaces is small distorted. To get a more accurate result, we should carefully optimize the location of nodes and the shape parameter of RBF.

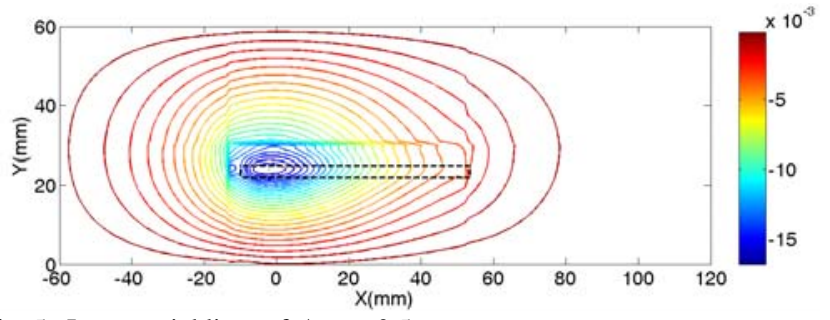

Fig. 5. Isopotential lines of $A_{e}$ at $t=0.5 \mathrm{~ms}$.

\section{CONCLUSION}

DDM combined RBF collocation method is first implemented to solve moving conductor eddy current magnetic field problems in this paper. With this novel method, the computational capacity could be greatly reduced compared with the normal RBF model. This offers us a useful tool to solve electromagnetic systems involving conductor movement with multi-materials, large size and complex shapes.

\section{ACKNOWLEDGMENT}

This work was supported by the National Natural Science Foundation of China (NSFC) under Grant 50877029 and the Specialized Research Fund for the Doctoral Program of Higher Education (SRFDP) under Grant 20100142110004.

\section{REFERENCES}

[1] B. Davat, Z. Ren and M. Lajoie-Mazenc, "The movement in field modeling," IEEE Trans. Magn., vol. MAG-21, no. 6, pp. 2296-2298, Nov. 1985.

[2] H. C. Lai, D. Rodger, and P. J. Leonard, "Coupling meshes in 3D problems involving movements,” IEEE Trans. Magn., vol. 28, no. 2, pp. 1732-1734, Mar. 1992.

[3] K. Yamazaki, S. Watari, and A. Egawa, "Adaptive finite element meshing for eddy current analysis of moving conductor," IEEE Trans. Magn., vol. 40, no. 2, pp. 993-996, Mar. 2004.

[4] K. Tani and T. Yamada, "Dynamic analysis of linear actuator taking into account eddy currents using finite element method and 3-D mesh coupling method," IEEE Trans. Magn., vol. 35, no. 3, pp. 1785-1788, May 1999.

[5] S. Kurz, J. Fetzer, G. Lehner, and W. M. Rucker, “A novel formulation for 3-D eddy current problems with moving bodies using a Lagrangian description and BEM-FEM coupling," IEEE Trans. Magn., vol. 34, no. 5, pp. 3068-3073, Sep. 1998.

[6] P. Ying, J.J. Ruan, Y. Zhang and Y. Gan, "A Composite Grid Method for Moving Conductor Eddy-Current Problem,” IEEE Trans. Magn., vol. 43, no. 7, pp. 3259-3265, Jul. 2007.

[7] Y. Zhang, K.R. Shao, Y.G. Guo, J.G. Zhu, and J.D. Lavers, "Multiquadrics collocation method for transient eddy current problems," IEEE Trans. Magn., vol. 42, no. 10, pp. 3183-3185, Oct. 2006.

[8] G.Y. Yang, Y. Zhang, G. Lei, K.R. Shao, Y.G. Guo, J.G. Zhu and J.D. Lavers, "A novel superposition RBF collocation method to solve moving conductor eddy current problems," IEEE Trans. Magn., vol. 45, no. 10, pp. 3977-3980, Oct. 2009.

[9] Y. Duan, P.F. Tang, T.Z. Huang and S.J. Lai, "Coupling projection domain decomposition method and Kansa's method in electrostatic problems," Computer Physics Communications, vol.180, no. 2, pp. 209214, Feb. 2009.

[10] G.Y. Yang, X.M. Chen, G. Lei, K.R. Shao, Y.G. Guo, J.G. Zhu and J.D. Lavers, "Application of meshless collocation method to solve eddy current magnetic field problems involving conductor movement," 2010 14th Biennial IEEE Conference on Electromagnetic Field Computation (CEFC 2010), 2010.

[11] P.J. Leonard, H.C. Lai, G. Hainsworth, D. Rodger and J.F. Eastham, "Analysis of the performance of tubular pulsed coil induction launchers,” IEEE Trans. Magn., vol. 29, no. 1, pp. 686-690, Jan. 1993. 